\title{
Intelligent wachsen. Die grüne Revolution
}

\author{
RALF FÜCKS
}

Vierzig Jahre nach dem Donnerschlag, den der Club of Rome mit seinem Report zu den „Grenzen des Wachstums“ auslöste, erleben wir eine Renaissance der Wachstumskritik. Der Ruf nach „Wohlstand ohne Wachstum“ findet Resonanz in allen politischen Richtungen, von links bis konservativ. So anregend diese Debatte auch sein mag, grenzt sie doch an Weltflucht. Sie blendet schlicht aus, dass wir mit einer stürmischen Wachstumsperiode der Weltwirtschaft konfrontiert sind, angetrieben von den Wünschen und Ambitionen von Milliarden Menschen, die ein besseres Leben für sich und ihre Kinder anstreben. Nullwachstum ist keine reale Option. Das gilt zumindest für den allergrößten Teil der Welt, der sich gerade im Aufbruch in die industrielle Moderne befindet. Es gilt aber, bei Lichte besehen, auch für „Old Europe“.

Die globale Wirtschaftsleistung wird sich in den kommenden 20 bis 25 Jahren glatt verdoppeln. Das ist eine gute und eine alarmierende Nachricht zugleich. Gut, weil damit sinkende Kindersterblichkeit, längere Lebenserwartung, bessere Bildung und sozialer Aufstieg in großem Stil einhergehen. Alarmierend, weil eine Verdoppelung des Ressourcenverbrauchs und der Emissionen auf einen ökologischen Super-Gau hinausliefe. Das alte, ressourcenfressende und energieintensive Wachstumsmodell ist nicht steigerbar. Wenn also ein bloßes „Weiter so" in ein globales Desaster mündet und der Ruf nach einem „Ende des Wachstums“ ins Leere geht, was ist dann die Alternative? Die zentrale Herausforderung der kommenden Jahrzehnte ist der Übergang zu einer Produktionsweise, die wachsenden Wohlstand ermöglicht, ohne die natürlichen Lebensgrundlagen zu ruinieren.

Auch in Europa schwimmt die Mehrheit der Bevölkerung keineswegs im Wohlstand. Im Gefolge der Finanzkrise ist die Jugendarbeitslosigkeit auf Rekordstände geklettert, viele Millionen Menschen sind verarmt. Damit alle ein Leben auf der Höhe ihrer Möglichkeiten führen können, braucht es nicht nur eine gerechtere Verteilung des Reichtums, sondern eine prosperierende Wirtschaft mit einer starken industriellen Basis.
Der Weg aus der Krise führt über eine Innovationsoffensive, die Europa an die Spitze der ökologischen Modernisierung hievt. Unser Kontinent hat das wissenschaftliche und industrielle Potenzial, zum Vorreiter der grünen industriellen Revolution zu werden. Die „Energiewende“ ist dafür ein Referenzprojekt: mit ihr können wir demonstrieren, dass der Abschied von der fossil-nuklearen Energieversorgung ein ökonomisches Erfolgsmodell sein kann. Nur dann wird sie auch zum Modell für die aufstrebenden Gesellschaften Asiens, Afrikas und Lateinamerikas.

\section{Entkoppelung von Wertschöpfung und Naturverbrauch}

Kern der grünen industriellen Revolution ist die Entkopplung von wirtschaftlicher Wertschöpfung und Naturverbrauch. Die kategorische Behauptung, das sei nicht möglich, ist so plausibel wie die Aussage „Der Mensch kann nicht fliegen“. Wachsender Wohlstand für Milliarden Menschen bei gleichzeitig sinkendem Umweltverbrauch erfordert erstens die weitgehende Dekarbonisierung der Ökonomie. Das gilt für den Energiesektor ebenso wie für die Chemieindustrie, für Transport, Städtebau und Landwirtschaft. Ein zweites Innovationsfeld ist die Steigerung der Ressourceneffizienz. Die Formel der Zukunft heißt: aus Weniger mehr machen. In den letzten 150 Jahren hat der Kapitalismus vor allem die Arbeitsproduktivität in immer neue Höhen getrieben. Künftig geht es darum, vor allem die Ressourcenproduktivität zu steigern. Ein dritter qualitativer Sprung ist der Übergang von linearen Produktketten zu vernetzten Stoffkreisläufen: Alle Reststoffe werden zum Ausgangspunkt neuer Produktionsprozesse. Künftig dürfen nur noch solche Stoffe in Verkehr gebracht werden, die entweder vollständig in den biologischen oder in den industriellen Kreislauf zurückgeführt werden können.

Die ökologische Transformation des Kapitalismus ist ein gewaltiges Innovations- und Investitionsprogramm. Es geht um die Erneuerung des gesamten industriellen Apparats und der öffent- lichen Infrastruktur. Zugleich sind die Investitionen von heute das Wachstum von morgen. In einer schrumpfenden Ökonomie schrumpfen auch die Investitionen, die Innovationsrate sinkt. Anhänger der „Entschleunigung“ mögen das begrüßen. Aber aus ökologischer Sicht wäre das fatal. Der Wettlauf mit der Klimakrise erfordert vielmehr eine Beschleunigung des strukturellen Wandels. Das impliziert steigende Investitionen und ein höheres Innovationstempo, also das Gegenteil einer Postwachstums-Strategie.

\section{Grenzen des Wachstums, Wachstum der Grenzen}

Die menschliche Zivilisation hängt an einem halbwegs stabilen Klima, an der Fruchtbarkeit landwirtschaftlicher Böden und an intakten Wasserkreisläufen. Überschreiten wir die Belastungsgrenzen der Ökosysteme, drohen schwere Krisen und Verwerfungen. Insofern gibt es sehr wohl ökologische Grenzen des Wachstums. Der springende Punkt ist, dass aus diesen "roten Linien“ keine fixen Grenzen für Produktion und Konsum folgen. Was dem Menschen auf unserem Planeten möglich ist, wird nicht in erster Linie von geophysikalischen Faktoren bestimmt. Unsere allerwichtigste Ressource heißt Kreativität. Dazu gehört auch die Fähigkeit, Knappheitskrisen durch Innovationen zu überwinden. Auch der Faktor Energie ist nicht begrenzt. Das Kraftwerk Sonne ist eine nahezu unerschöpfliche Energiequelle. Dabei geht es nicht nur um die Gewinnung von Strom und Wärme aus Sonnenenergie, sondern um die technische Simulation der Photosynthese - die Umwandlung von Sonnenlicht, Wasser und $\mathrm{CO}_{2}$ in biochemische Energie.

Auf Erfindungsreichtum und Unternehmergeist als Produktivkraft zu setzen, enthebt uns nicht von persönlicher Verantwortung. Es ist gut und richtig, weniger Fleisch zu essen, mit Rad oder Bahn zu fahren und keine Produkte zu kaufen, für die Menschen geschunden oder Regenwälder abgeholzt werden. Jedem steht es frei, das "gute Leben" in einem Mehr an freier Zeit und sozialen Beziehungen statt in einer Steigerung von Einkom- 
men und Konsum zu suchen. Aber ein nüchterner Blick auf die Größe der ökologischen Herausforderung zeigt, dass sie mit dem Appell zur Genügsamkeit nicht zu lösen ist. „Weniger vom Gleichen “ ist nicht genug. Ohne groß angelegte Energie- und Effizienzrevolution werden wir den Wettlauf mit dem Klimawandel nicht gewinnen. Soziale Innovationen wie die neue Ökonomie des Teilens oder die „Slow Food“-Bewegung sind wichtige Treiber des Wandels. Hier wie dort geht es aber nicht um die Abkehr vom Lebensstil der Moderne, sondern um einen reflektierten Hedonismus.

Die ökologische Transformation des Kapitalismus ist keine Gesundbeterei. Wer genauer hinschaut, entdeckt überall Anzeichen des Wandels. Das gilt für das rasante Wachstum erneuerbarer Energien, die Einführung betrieblicher Umweltund Sozialbilanzen oder die Kooperation zwischen Unternehmen und Zivilgesellschaft bei der Zertifizierung nachhaltiger Produkte. Trotz aller Exzesse der Finanzindustrie zeichnet sich ein neuer Trend zur moralischen Aufladung der Ökonomie ab. Skandale um unmenschliche Arbeitsbedingungen, ökologischen Raubbau oder Steuerflucht von
Konzernen führen zur Re-Regulierung einer aus dem Ruder gelaufenen Wirtschaft. Parallel ist in vielen Betrieben ein Kulturwandel im Gang. In postmaterialistischen Gesellschaften gewinnt das „moralische Kapital“ von Unternehmen wachsende Bedeutung für ihren wirtschaftlichen Erfolg. Skandalprävention durch Beachtung sozialer und ökologischer Mindeststandards ist betriebswirtschaftlich ebenso rational wie der effiziente Umgang mit Ressourcen. Nachhaltige Investmentfonds sind im Kommen. Gleichzeitig beobachten wir eine Renaissance der gemeinnützigen Ökonomie. Non-Profit-Unternehmen und Open-SourceProjekte sind Trend. Unter jungen Leuten ist das Prinzip „Nutzen statt Besitzen“ populär, Tauschportale im Internet florieren und selbst die großen Autokonzerne bauen Car-Sharing-Projekte auf.

Wird also alles gut? Sicher nicht, und schon gar nicht von selbst. Märkte, Wettbewerb und Unternehmertum sind als innovative Suchverfahren unverzichtbar. Um aber mehr Nachhaltigkeit in die Marktwirtschaft zu bringen, braucht es einen politisch-rechtlichen Ordnungsrahmen. Die sukzessive Verteuerung des Ressourcenver- brauchs, ein effektiver $\mathrm{CO}_{2}$-Emissionshandel, ambitionierte Effizienzstandards, transparente Produktinformationen, die Verpflichtung der Hersteller auf die Rücknahme ausrangierter Geräte und Fahrzeuge, eine ökologisch ausgerichtete Forschungs- und Technologiepolitik wirken als Hebel in diese Richtung. Aber auch der Staat allein wird es nicht richten. Ebenso nötig sind eine kritische Öffentlichkeit, aufgeklärte Konsumenten und starke zivilgesellschaftliche Akteure, die Alarm schlagen und Handlungsdruck aufbauen können. In der Kombination dieser Faktoren liegt die Chance, die Krise der Moderne mit den Mitteln der Moderne zu bewältigen.

\section{AUTOR}

RALF FÜCKS, Diplom-Sozialwissenschaftler, Vorstand der Heinrich-Böll-Stiftung und Autor von „Intelligent wachsen. Die grüne Revolution“ (Berlin 2013).

fuecks@boell.de 